\title{
Atomic motion in ferromagnetic break junctions
}

To the Editor - Sokolov et al. ${ }^{1}$ report measurements of the conductance of ferromagnetic point contacts at room temperature as a function of the angle of an applied magnetic field. They find abrupt steps in conductance, of the order of $e^{2} / h$ in size, at particular field angles, which they ascribe to an intrinsic electronic mechanism associated with the opening and closing of discrete quantum channels in the point contact. Here we show that the angle of an applied magnetic field can have a surprisingly strong effect in causing sudden changes to the configuration of atoms in some nanoscale ferromagnetic contacts, thereby inducing reproducible steps in the electrical conductance as a function of magnetic-field angle. Even at a temperature of $4.2 \mathrm{~K}$ the atoms are not always frozen in place. On this basis, we suggest that atomic rearrangements cannot be ruled out as a possible explanation for the results given by Sokolov and colleagues.

We have performed conductance measurements on magnetic point contacts as a function of both field angle and time, at temperatures of $4.2 \mathrm{~K}$ and above. The field angle was adjusted using a multiple-coil superconducting magnet. We investigated $\mathrm{Ni}$ and permalloy $\left(\mathrm{Ni}_{80} \mathrm{Fe}_{20}\right)$ contacts formed by electronbeam lithography and evaporation onto oxidized silicon substrates, followed by controlled electromigration to narrow the contacts to near-atomic cross-sections ${ }^{2}$. In accord with previous experiments on point contacts made from other metals ${ }^{3,4,5}$, our measurements of conductance as a function of time reveal two-level conductance fluctuations in all samples at temperatures above several tens of $\mathrm{K}$ (and occasionally even at $4.2 \mathrm{~K}$ ), owing to the motion of atoms or small groups of atoms between metastable arrangements. Magnetic point contacts at room temperature are therefore not static devices, but contain atoms or collections of atoms fluctuating between different positions over a broad range of timescales.

In measurements of conductance as a function of the angle of an applied magnetic field, in approximately $10 \%$ of samples we have observed abrupt steps at particular field angles, similar to the results of Sokolov and co-workers. Figure 1a shows an example of a $\mathrm{Ni}$ device; we have observed qualitatively similar behaviour in permalloy devices
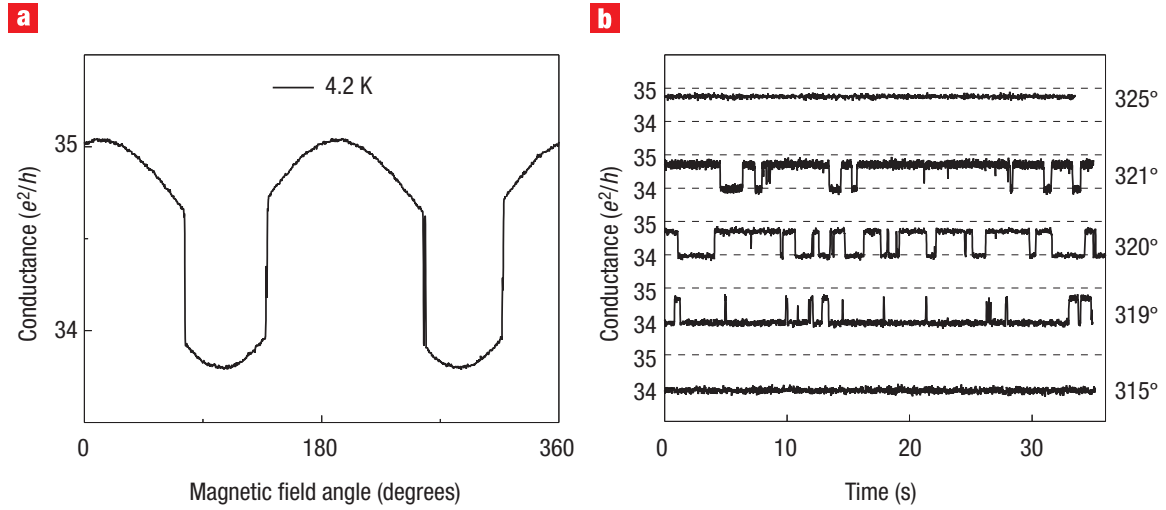

Figure 1 Abrupt conductance changes in a nanoscale Ni contact at $4.2 \mathrm{~K}$. a, Conductance as a function of magnetic field angle, for a field magnitude of $800 \mathrm{mT}$. The field is rotated in the sample plane. $\mathbf{b}$, Conductance as a function of time at several fixed field angles, for the same sample as in $\mathbf{a}$. At field angles in the vicinity of the conductance steps in a, we observe two-level conductance switching owing to atomic motion.

as well. In all cases where we observe these abrupt steps, measurements as a function of time at fixed field angle near the transition point reveal twolevel fluctuations with size equal to the conductance step as a function of angle (Fig. 1b). The duty cycle of the time-dependent fluctuations varies continuously from being in the highconductance state $0 \%$ of the time to $100 \%$ of the time over a narrow range of field angles in the transition region, demonstrating that the conductance change as a function of angle is a consequence of the atomic motion - if the effects of any pre-existing atomic motion were merely amplified in the transition region by modulating the opening and closing of a discrete quantum channel then the duty cycle would not change. We conclude that, even in magnetic point contacts designed to minimize magnetostriction and magnetostatic forces, the angle of an applied magnetic field can strongly affect the stability of atoms in the contact region and produce abrupt reproducible steps in conductance versus field angle owing to atomic reconfigurations.

The observation of Sokolov et al. that their conductance steps occur at slightly different field angles upon repeated measurements is also explained more easily as a consequence of relatively slow atomic motion rather than by an intrinsic electronic mechanism, as thermal fluctuations in the population of electronic states should fluctuate much more quickly than the millisecond timescales of their experiment.

\section{S.-F. Shi and D. C. Ralph ${ }^{*}$ \\ Laboratory of Atomic and Solid State Physics, Cornell University, Ithaca, New York 14853 USA *e-mail:ralph@ccmr.cornell.edu}

\section{References}

1. Sokolov, A., Zhang, C., Tsymbal, E. Y., Redepenning, J. \& Doudin, B. Nature Nanotech. 2, 171-175 (2007).

2. Bolotin, K. I., Kuemmeth, F., Pasupathy, A. N. \& Ralph, D. C. Nano Lett. 6, 123-127 (2006).

3. Ralls, K. S., Ralph, D. C. \& Buhrman, R. A. Phys. Rev. B 40, 11561-11570 (1989).

4. Holweg, P. A. M., Caro, J., Verbruggen, A. H. \& Radelaar, S. Phys. Rev. B 45, 9311-9319 (1992).

5. Agrait, N., Levy Yeyati, A. \& van Ruitenbeek, J. M. Phys. Rep. 377, 81-279 (2003).

Authors' response - In their comment ${ }^{1}$, Shi and Ralph emphasize that conductance of $\mathrm{Ni}$ point contacts produced by an electromigration technique can occasionally exhibit two-level fluctuations (TLF), mimicking the discrete steps in the conductance of the point contacts as a function of the applied magnetic-field direction. They explain the nature of these steps in terms of atomic motion rather than intrinsic electronic effect. We emphasize in this reply that our results ${ }^{2}$, obtained on a different magnetic material synthesized by a different method, do not show any data confirming their hypothesis.

Ballistic anisotropic magnetoresistance (BAMR) was observed on Co point contacts electrochemically grown between $\mathrm{Au}$ or Ni electrodes. No evidence of 